\title{
Prediction of pancreatic cancer risk and therapeutic response with next-generation sequencing
}

\author{
Ioannis D Kyrochristos ${ }^{1,2}$, Demosthenes E Ziogas ${ }^{1,3}$, Georgios K Glantzounis ${ }^{2}$ \& Dimitrios H \\ Roukos ${ }^{*}, 1,2,4$ \\ ${ }^{1}$ Centre for Biosystems \& Genome Network Medicine, Ioannina University, loannina, 45110, Greece \\ ${ }^{2}$ Department of Surgery, loannina University Hospital, loannina, 45110, Greece \\ ${ }^{3}$ Department of Surgery, 'G. Hatzikosta' General Hospital, loannina, 45001, Greece \\ ${ }^{4}$ Department of Systems Biology, Biomedical Research Foundation of the Academy of Athens (BRFAA), Athens, 11527, Greece \\ * Author for correspondence: Tel.: +302651005572; droukos@uoi.gr
}

First draft submitted: 18 September 2017; Accepted for publication: 20 September 2017; Published online: 13 December 2017

Keywords: biomarkers $\bullet$ intratumor heterogeneity $\bullet$ pancreatic cancer $\bullet$ prediction $\bullet$ RNA sequencing $\bullet$ wholegenome sequencing

The overall 5-year survival rates of $8 \%$ [1] reflect the grim prognosis of patients with pancreatic ductal adenocarcinoma (PDA), which is at least partially explained by the unfruitful efforts to identify prognostic and predictive biomarkers toward tailored and optimized therapeutic interventions. Extensive heterogeneity at the level of molecular-signaling pathways [2,3], high metastatic capacity and significant therapeutic resistance suggest the need for a strategic shift to screening and early detection, as well as the development of more effective, novel systemictargeted therapies. This opinion article summarizes the potential of next-generation sequencing (NGS) and the emerging challenges in completing the catalog of cancer-driver genes (CDGs) for pancreatic cancer, detecting high-risk individuals through intensive screening and predicting drug response.

Nevertheless, a recent, large-scale study by Bailey et al. [4], implementing whole-genome sequencing (WGS) and RNA sequencing (RNAseq) in an outstanding innovative design, has finally demonstrated significant clinical implications.

The development of robust biomarkers is crucial to personalize and improve poor outcomes in patients with pancreatic cancer. However, no progress has been made in biomarker-based individualized preventive and therapeutic approaches, with 5-year survival rates remaining low, even in the early, localized disease stages [1].

Despite the guidelines composed by national and international organizations prompting PDA patient enrollment in clinical trials, research has featured very slow progress in this area. More specifically, the lack of both effective targeted drugs and biomarkers-directed agents raises questions on whether a strategy of conventional clinical trial designs and traditional technology is able to alter the grim survival of PDA [5].

The advent of NGS technologies about a decade ago and the development of umbrella and basket clinical trial designs integrating NGS [3] pave new avenues toward the precise biomarker-based prevention and treatment of pancreatic cancer. To this direction, recent large-scale genomic studies combining WGS and RNAseq have provided promising data and clinical implications on PDA prognosis and molecular classification [4,6].

\section{Precise prediction: an unmet clinical need}

Several environmental risk factors have been associated with pancreatic cancer. These include smoking, heavy alcohol consumption and chronic pancreatitis, exposure to specific chemicals and heavy metals, increased BMI and decreased physical activity, increased consumption of red and processed meat and dairy products, hepatitis B infection, diabetes mellitus, prediabetes, insulin and sulfonylureas [5]. It is worth noting that PDA patients with diabetes have a lower overall survival, compared with nondiabetics [7].

Apart from the environmental effect on pancreatic tumorigenesis, pancreatic cancer has a familial component in $10 \%$ of cases and familial PDA excess, as well as earlier onset, increases the risk of cancer [8]. Nevertheless,

Future Medicine 
the genetic basis of familial PDA remains unclear in approximately 80\% of cases [8]. However, several syndromes of familial cancer have been associated with an increased risk of PDA, including Peutz-Jeghers syndrome, familial pancreatitis, familial melanoma-pancreatic cancer syndrome, Lynch syndrome and hereditary breast-ovarian cancer syndrome (especially BRCA2-mutated) and more than ten cancer predisposition genes (CPGs) have been identified for pancreatic cancer [5]. Furthermore, two types of premalignant tumors have been identified; mucinous cystic neoplasms and intraductal papillary mucinous neoplasms. The former and intraductal papillary mucinous neoplasms of the main duct bare increased risk of malignancy at around 15 and 62\%, respectively [9], and current guidelines recommend resection of these lesions [5].

Screening for PDA is generally not indicated for asymptomatic individuals [5]. The Cancer of the Pancreas Screening consortium recommends the use of endoscopic ultrasound (EUS) and/or magnetic resonance imaging/cholangiopancreatography (MRI/MRCP) for high-risk individuals, defined as first-degree relatives of patients with familial PDA, carriers of $p 16$ or BRCA2 mutations with an affected first-degree relative, patients with Peutz-Jeghers syndrome or patients with Lynch syndrome and one affected first-degree relative [10]. However, further studies and evidence are required to determine the optimal management in case of positive findings, the appropriate age to begin screening and the follow-up intervals. Moreover, the tumor marker CA 19-9 could harbor screening, diagnostic, staging, prognostic and predictive significance [11]. Nevertheless, current guidelines recommend measurement before surgery, before adjuvant treatment and during surveillance to follow therapeutic response, while they discourage its use for screening, due to its low-positive predictive value [5].

In summary, no screening strategy exists for the early detection of PDA, such for breast, prostate and colorectal cancer [5]. Similarly, no high-penetrance genes have been identified, in contrast to well-known hereditary cancer syndromes (BRCA1/2 in breast-ovarian, mismatch-repair genes in hereditary nonpolyposis colorectal and endometrial cancer and $\mathrm{CDH} 1$ for diffuse gastric-breast cancer). For these syndromes, risk-reducing surgery is indicated [5]. Exciting biomedical research implementing NGS is underway to identify novel CPGs for future screening and effective prevention.

\section{Genome sequencing technologies \& potential biomarkers}

Recently, pancreatic cancer has been an intriguing research subject and significant effort has been put into the identification of robust biomarkers and the development of a valid molecular classification with clinical relevance [12]. To this end, mismatch-repair and homologous recombination-repair deficiency have been identified as eventual biomarkers for PDA with increased mutational burden. Their clinical significance lies in the potential of patient selection for future clinical trials on targeted immune inhibition, based on recent data supporting the increased efficacy of immune checkpoint inhibitors in highly mutated cancers [6].

Two large-scale studies have attempted molecular PDA subtyping, based on genomic and transcriptomic characteristics. First, Connor et al., while noting the potential role of immune checkpoint inhibition in specific PDA subtypes, concluded that their classification featured no clinical implications [13]. On the other hand, Bailey et al. identified and validated four major pancreatic cancer subclasses, which were associated with distinct histopathologic and clinical characteristics. More specifically, the squamous subtype, transcriptionally identified in other tumor types as well, correlated with increased metastatic potential and worse prognosis, through upregulation of specific metastasis-related genes. Finally, this is yet another study supporting the role of immune inhibitors in pancreatic cancer, highlighting the potential targetability of the immunogenic PDA subclass, characterized by CTLA4 and PD1 pathway upregulation [4].

The composition of a novel PDA molecular classification with clinical significance [4] undisputedly opens new preventive and therapeutic horizons. Nevertheless, several challenges remain unsolved. Those include the completion of the CDG and the CPG catalogs, as well as overcoming the dynamic emergence of tumor heterogeneity and therapeutic resistance, through innovative research directions.

\section{Future perspective \& conclusion}

The integration of NGS into appropriately designed studies points a new direction to ultimately develop valid prognostic, predictive and therapeutic biomarkers. The excellent classification of pancreatic cancer based on the coupled utilization of WGS and RNAseq [4] prompts the conduction of further clinical trials examining the preventive and therapeutic efficacy of genome-based analyses.

The basic research aim to complete the list of CDGs and CPGs with whole-exome sequencing (WES) and WGS raises the opportunity not only to discover novel oncotargets but also to use them at very low cost for 
targeted next-generation sequencing (tNGS). Moreover, the completion of these catalogs raises new opportunities for screening high-risk individuals who meet the criteria of both exposure to established environmental factors and harboring identified CPGs. In these individuals, early disease detection could be achieved by EUS, CT or MRI, which require validation by clinical trials on their clinical utility. However, the establishment of new CDGs and $\mathrm{CPGs}$ in the available list of sporadic and inherited mutations for subsequent $\mathrm{tNGS}$ analyses requires the conduction of large-scale WES and WGS studies to capture the extensive genetic and genomic tumor heterogeneity [14]. For instance, the list of CDGs and CPGs has recently expanded to six new genes for breast cancer, by two large studies, employing WES on 892 breast tumors (one novel gene) and WGS on 560 breast cancers (five novel genes) [14,15].

Although conventional, single-biopsy WES/WGS can often provide clinical implications, overcoming therapeutic resistance requires breakthrough NGS methods. To help us understand the role of the dynamic subclonal evolution in tumor heterogeneity, inherent and acquired therapeutic resistance, two novel methods have been developed. First, the conduction of NGS on multiple, spatially discrete tumor samples (multiregional NGS - MR-NGS) enables the assessment of intratumor heterogeneity (ITH) in the primary tumor [16]. Evidence on dynamics of genomic clone evolution according to the Darwinian theory [17] opens new clinical horizons for patients treated with neoadjuvant treatment. A recent study exploring ITH with MR-NGS before and after systemic therapy has provided data supporting the development of resistant subclones in response to treatment [18,19].

Second, the noninvasive identification of circulating genomic subclones (cGSs) by sequencing plasma cellfree DNA (cfDNA-NGS), before and after therapeutic interventions, as well as over the disease course, offers unprecedented clinical potential. The clinical applications of serial cfDNA-NGS include the appropriate design of primary systemic treatment by comparing ITH with cGSs and providing the optimal drug combination for every individual patient [20]. Moreover, repeated cGS assessment during follow-up could be used to monitor patients at high risk of relapse [21]. Indeed, several studies have revealed the ability of cfDNA-NGS to predict recurrence, approximately 6 months before the clinical diagnosis by CT scanning [22]. Added to that, cfDNA further provides the opportunity for noninvasive diagnosis of PDA. Several cfDNA analyses utilizing tNGS have demonstrated this potential utility of liquid biopsies [23,24]. According to current guidelines, no histopathological diagnosis is suggested before surgical resection in most cases, while invasive EUS-fine-needle aspiration (EUS-FNA) biopsy is associated with complications [5]. Should the validity and accuracy of cGS identification as a prognostic biomarker be confirmed in large validation trials, the unprecedented power to prevent relapse by early cGS targeting with existing or novel targeted drugs, paves new avenues for future research.

In summary, biomarker-directed personalized prevention and treatment represents a compelling research prospect, to improve the grim oncological outcomes of PDA patients. Concerning early detection and prevention, completion of the CDG and CPG catalog, in conjunction with the identification of environmental risk factors and the understanding of the extensive molecular landscape and environment-gene interactions, forms the basis of effective screening and prevention in high-risk individuals. As for treatment, new designs of clinicogenomic studies integrating WGS, RNAseq, MR-NGS and serial cGS identification raise great expectations toward the precise and personalized treatment of individual patients with pancreatic cancer.

\section{Financial \& competing interests disclosure}

The authors have no relevant affiliations or financial involvement with any organization or entity with a financial interest in or financial conflict with the subject matter or materials discussed in the manuscript. This includes employment, consultancies, honoraria, stock ownership or options, expert testimony, grants or patents received or pending, or royalties.

No writing assistance was utilized in the production of this manuscript.

\section{References}

1. Siegel RL, Miller KD, Jemal A. Cancer statistics, 2017. CA Cancer J. Clin. 67(1), 7-30 (2017).

2. Vogelstein B, Papadopoulos N, Velculescu VE, Zhou S, Diaz LA Jr, Kinzler KW. Cancer genome landscapes. Science 339(6127), 1546-1558 (2013).

3. Biankin AV, Piantadosi S, Hollingsworth SJ. Patient-centric trials for therapeutic development in precision oncology. Nature 526(7573), 361-370 (2015).

4. Bailey P, Chang DK, Nones K et al. Genomic analyses identify molecular subtypes of pancreatic cancer. Nature 531(7592), 47-52 (2016).

5. National Comprehensive Cancer Network, Inc. (2017). www.nccn.org/

6. Humphris JL, Patch AM, Nones K et al. Hypermutation in pancreatic cancer. Gastroenterology 152(1), 68.e62-74.e62 (2017). 
7. Raghavan SR, Ballehaninna UK, Chamberlain RS. The impact of perioperative blood glucose levels on pancreatic cancer prognosis and surgical outcomes: an evidence-based review. Pancreas 42(8), 1210-1217 (2013).

8. Hruban RH, Canto MI, Goggins M, Schulick R, Klein AP. Update on familial pancreatic cancer. Adv. Surg. 44, $293-311$ (2010).

9. Tanaka M, Fernandez-Del Castillo C, Adsay V et al. International consensus guidelines 2012 for the management of IPMN and MCN of the pancreas. Pancreatology 12(3), 183-197 (2012).

10. Canto MI, Harinck F, Hruban RH et al. International Cancer of the Pancreas Screening (CAPS) consortium summit on the management of patients with increased risk for familial pancreatic cancer. Gut 62(3), 339-347 (2013).

11. Morris-Stiff G, Taylor MA. Ca19-9 and pancreatic cancer: is it really that good? J. Gastrointest. Oncol. 3(2), 88-89 (2012).

12. Kyrochristos ID, Glantzounis GK, Ziogas DE et al. From clinical standards to translating next-generation sequencing research into patient care improvement for hepatobiliary and pancreatic cancers. Int. J. Mol. Sci. 18(1), pii: E180 (2017).

13. Connor AA, Denroche RE, Jang GH et al. Association of distinct mutational signatures with correlates of increased immune activity in pancreatic ductal adenocarcinoma. JAMA Oncol. 3(6), 774-783 (2017).

14. Lawrence MS, Stojanov P, Mermel CH et al. Discovery and saturation analysis of cancer genes across 21 tumour types. Nature 505(7484), 495-501 (2014).

15. Nik-Zainal S, Davies H, Staaf J et al. Landscape of somatic mutations in 560 breast cancer whole-genome sequences. Nature 534(7605), 47-54 (2016).

16. Jamal-Hanjani M, Wilson GA, McGranahan N et al. Tracking the evolution of non-small-cell lung cancer. N. Engl. J. Med. 376(22), 2109-2121 (2017).

17. Eirew P, Steif A, Khattra J et al. Dynamics of genomic clones in breast cancer patient xenografts at single-cell resolution. Nature 518(7539), 422-426 (2015).

18. Yates LR, Gerstung M, Knappskog S et al. Subclonal diversification of primary breast cancer revealed by multiregion sequencing. Nat. Med. 21(7), 751-759 (2015).

19. Murugaesu N, Wilson GA, Birkbak NJ et al. Tracking the genomic evolution of esophageal adenocarcinoma through neoadjuvant chemotherapy. Cancer Discov. 5(8), 821-831 (2015).

20. Roukos DH. Spatiotemporal diversification of intrapatient genomic clones and early drug development concepts realize the roadmap of precision cancer medicine. Drug Discov. Today 22(8), 1148-1164 (2017).

21. Frenel JS, Carreira S, Goodall J et al. Serial next-generation sequencing of circulating cell-free DNA evaluating tumor clone response to molecularly targeted drug administration. Clin. Cancer Res. 21(20), 4586-4596 (2015).

22. Heitzer E, Ulz P, Belic J et al. Tumor-associated copy number changes in the circulation of patients with prostate cancer identified through whole-genome sequencing. Genome Med. 5(4), 30 (2013).

23. Takai E, Totoki $\mathrm{Y}$, Nakamura $\mathrm{H}$ et al. Clinical utility of circulating tumor DNA for molecular assessment in pancreatic cancer. Sci. Rep. 5, 18425 (2015).

24. Zill OA, Greene C, Sebisanovic D et al. Cell-free DNA next-generation sequencing in pancreatobiliary carcinomas. Cancer Discov. 5(10), 1040-1048 (2015). 